\title{
Effects of different nuclear evaluation data on the RMC keff calculation
}

\author{
Zhang W enxin ${ }^{1,2^{*}}$, Qiang shenglong ${ }^{1,2}, Y$ in qiang ${ }^{1,2}$ and Cui Xiantao ${ }^{1,2}$ \\ ${ }^{1}$ Nuclear Power Institute of China, Cheng Du, China \\ ${ }^{2}$ Science Technology on Reactor System Design Technology Laboratory, Cheng Du, China
}

\begin{abstract}
Neutron cross section data is the basis of nuclear reactor physical calculation and has a decisive influence on the accuracy of calculation results. AFA 3 Gassemble is widely used in nuclear power plants. CENA CE is an A CE format multiple-temperature continuous energy cross section library that developed by China Nuclear Data Centre. In this paper, we calculated the AFA $3 G$ assemble by RMC.We respectively used ENDF6.8/, ENDF/7 and CENACE data for calculation. The impact of nuclear data on RMC calculation is studied by comparing the results of different nuclear data.
\end{abstract}

\section{Introduction}

Neutron cross section library plays a key role in the core calculation. The ace format temperature dependent neutron cross section library for continuous energy M onte Carlo transport programs such as RMC and MCNP, which is processed by NJOY. The machining process includes resonance reconstruction, Doppler broadening, neutron thermalization. CENACE cross section library was obtained from the ENDF/B 7.1 evaluation database processed by the 401 using NJOY. In this paper, RMC is used for the modelling and calculation of AFA3G assemble. Then we analysis three groups data of $k_{\text {eff }}$ varies with time to sections the formatting shown in Table 2 should be used.

\section{Introduction of AFA3G assemble}

The AFA $3 G$ assemble is the third generation of advanced PWR fuel assemble designed by Framatome Each assemble contains 264 fuel rods and 25 guide tubes. AFA 3G assemble does not use a combination of discrete burnable poison and integrated burnable poison, but uses an integrated burnable poison design with Gadolinium. This paper calculates two assemblies, one is that the assemble contains 16 poison rods, and the other is the assemble without poison rod. B oth fuel assemblies do not take into account the grid, the structure of the assemble is shown in the figure 1 and figure 2 . The assemble has an enrichment of $3.1 \%$, with 264 fuel rods and 25 guide tubes.

\begin{tabular}{|c|c|}
\multicolumn{2}{|c|}{ Table 1.Parameters of AFA3G assemble } \\
\hline Parameter & Vlaue \\
\hline Assemble type & $17 \times 17$ \\
\hline Fuel pellet radius & $0.4096 \mathrm{~cm}$ \\
\hline $\begin{array}{c}\text { Fuel cladding } \\
\text { external diameter }\end{array}$ & $0.475 \mathrm{~cm}$ \\
\hline $\begin{array}{c}\text { Guide tube inside } \\
\text { diameter }\end{array}$ & $0.5605 \mathrm{~cm}$ \\
\hline $\begin{array}{c}\text { Guide tube external } \\
\text { diameter }\end{array}$ & $0.6225 \mathrm{~cm}$ \\
\hline Lattice interval & $1.26 \mathrm{~cm}$ \\
\hline
\end{tabular}

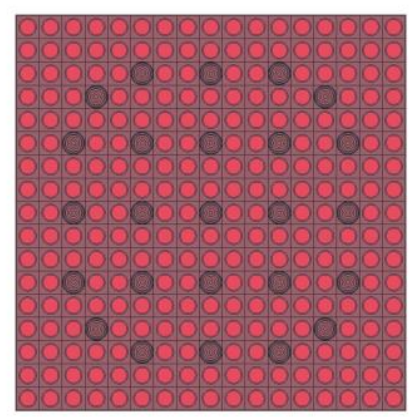

Fig. 1. A FA 3G 31000 assemble

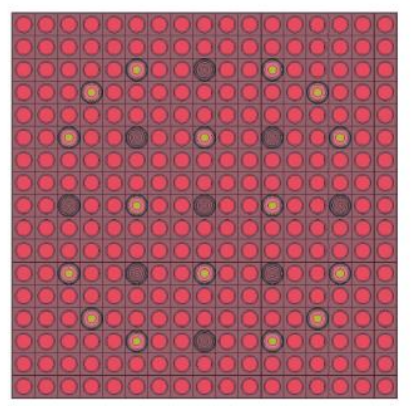

Fig. 2. AFA 3G 31016 assemble

\footnotetext{
Corresponding author: 18200228211@163.com
} 


\section{Introduction of RMC and cross section library}

We use RMC to model and calculate the assemble, and calculate the fuel burnup with a constant power of $40 \mathrm{M} \mathrm{W}$. The fuel burnup of the assemble is $90000 \mathrm{MWD} / \mathrm{TU}$, and burnup calculation is divided into 120 steps.

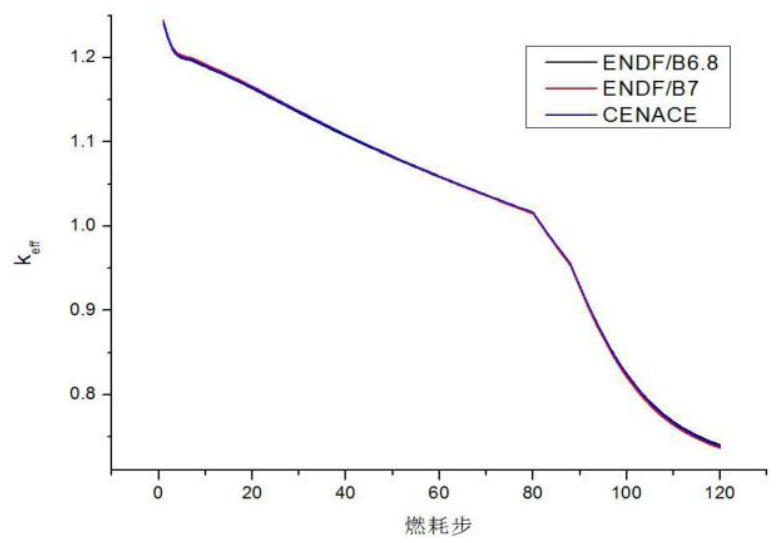

Fig. 3. Results of AFA 3G31000 A ssemble

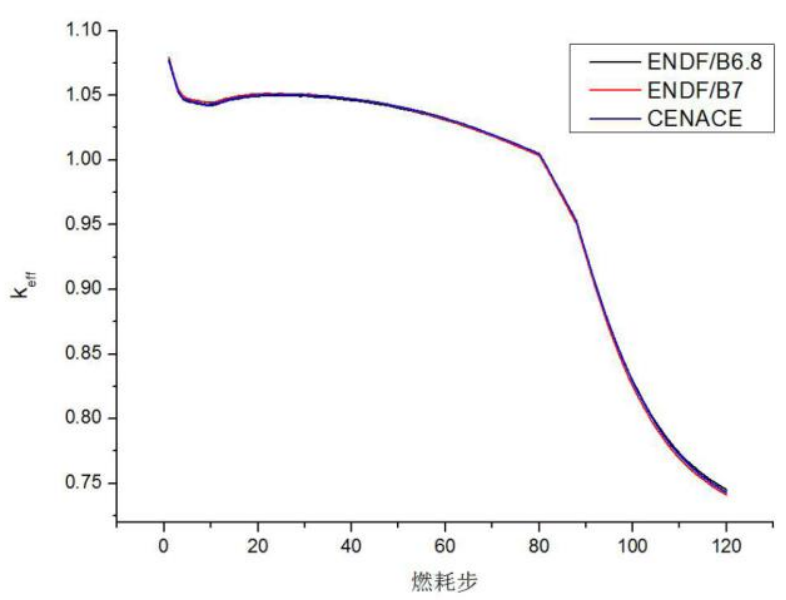

Fig. 4. Results of AFA 3G31016 A ssemble

As we can see from Figure 3 and figure 4, the results of two assemblies are not much different.

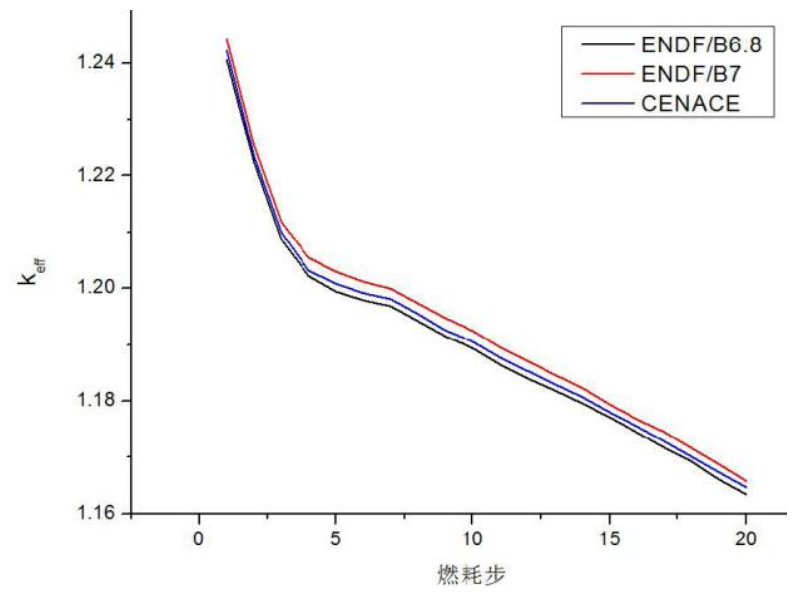

Fig. 5. Results of AFA 3G31000 assemble in first 20 steps

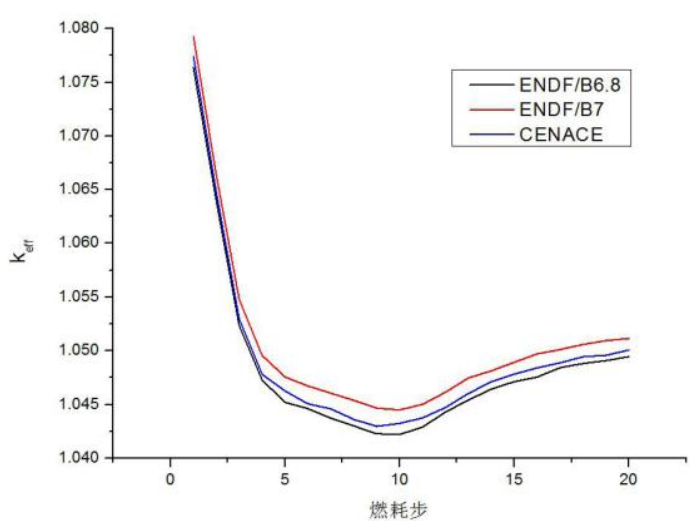

Fig.6. Results of AFA 3G31016 assemble in first 20 steps

Figure5 and Figure 6 show the calculation results of the first 20 steps, it can be seen that the $k_{\text {eff }}$ of ENDF/B 7 library is slightly higher than the other two libraries at the beginning of the burnup, and the $k_{\text {eff }}$ of ENDF/B6.8 library is the smallest.

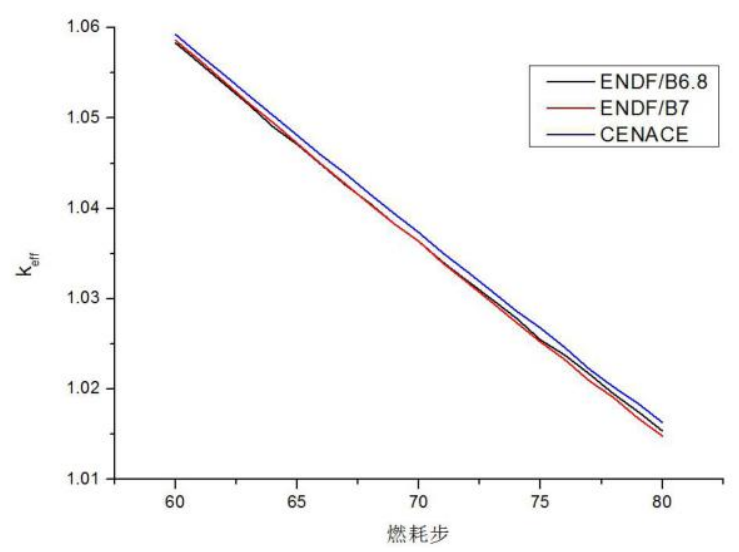

Fig.7. The results of AFA $3 G 31000$ assemble from steps 60 to 80

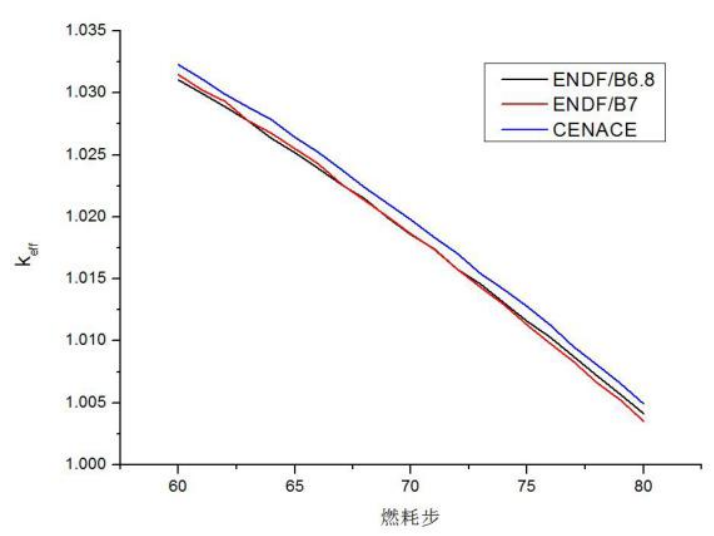

Fig.8. The results of A FA 3G 31016 assemble from steps 60 to 80

With the burnup, from steps 60 to 80 , results of ENDF/B7 and ENDF/6.8 library is basically same, results of CENACE library is slightly higher than the ENDF/B 7 and ENDF/6.8 library. 


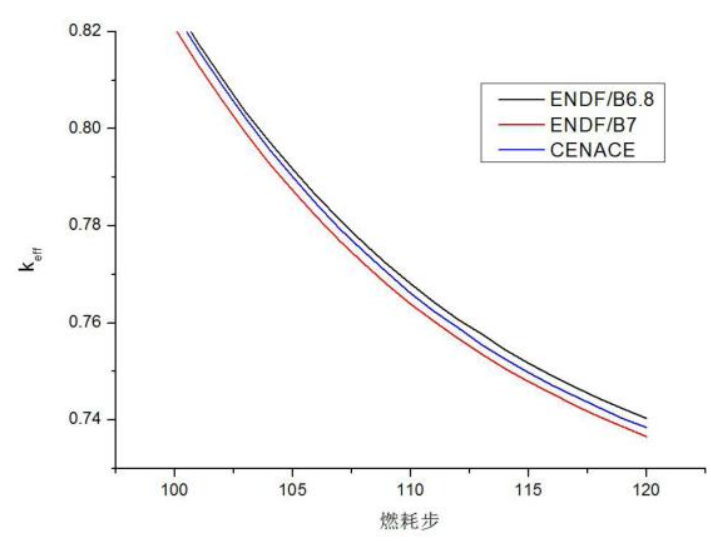

Fig.9. The results of A FA $3 G 31000$ assemble from steps 80 to 120

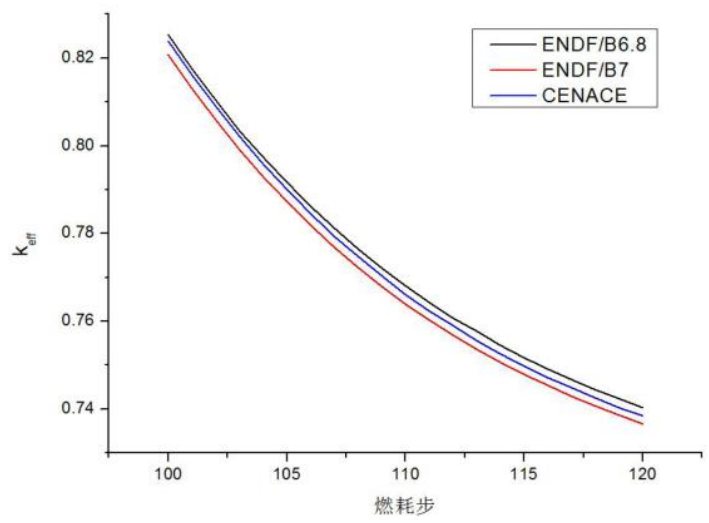

Fig.10. The results of A FA 3G 31016 assemble from steps 80 to 120

As can be seen from the above figure,at the end of period, ENDF/B 68 has the largest $k_{\text {eff }}$ and the ENDF/B 7 library has the smallest calculation results. with the burnup, compared with the CENACE, the $k$ calculated by the ENDF/B 7 library gradually decreases. The difference between the calculation results of the ENDF/B 68 and the ENDF/B 7 library is about -0.004 to 0.005 . The $k_{\text {eff }}$ calculated by ENDF/B7 is relatively small when in the deep burnup.

\section{Conclusion}

We used three cross section libraries to calculate AFA $3 G$ assemble. By comparing the calculation results, it is found that the calculation results of $k_{\text {eff }}$ under different library are very close. The $k_{\text {eff }}$ calculated by ENDF/B 7 is relatively small when in the deep burnup, and $k_{\text {eff }}$ of CENACE library is in between.

\section{References}

1. M cgreevy R L. RMC: progress, problems and prospects[] ]. N uclear Instruments \& M ethods in Physics Research, Section A, 354(1):1-16. (1995)

2. Liu S, Liang J, Wu Q, et al. BEA VRS full core burnup calculation in hot full power condition by
RM C code[] ]. A nnals of Nuclear Energy, 101:434446. (2017); L uigi T. De L uca, Propulsion physics (EDP Sciences, Les Ulis, 2009)

3. Guo J, Liu S, Shang X, et al. Coupled neutronics/ thermal-hydraulics analysis of a full PW R core using RM C and CTF[J]. A nnals of N uclear Energy, 109: 327-336 (2017).

4. Ouwen $Y$, Shichang $L, K$ an W. Research on RM C neutronics-thermal hydraulics coupling based on universal coupling methodology[]]. High Power L aser and Particle B eams(2017). 